\title{
Case report: mantle cell lymphoma, prolymphocytoid variant, with leukostasis syndrome
}

\author{
Marc D Smith ${ }^{1}$, Timothy P Singleton ${ }^{1}$, Savitha Balaraman ${ }^{2}$, Ishmael Jaiyesimi ${ }^{2}$, \\ Barbara O’Malley ${ }^{1}$, Abdul Al-Saadi ${ }^{3}$ and Joan C Mattson ${ }^{1}$ \\ ${ }^{1}$ Department of Clinical Pathology; ${ }^{2}$ Department of Hematology/Oncology and ${ }^{3}$ Department of Anatomic \\ Pathology, William Beaumont Hospital, Royal Oak, MI, USA
}

\begin{abstract}
A 76-year-old man presented with leukostasis syndrome, including oculodynia, blurred vision, and visual field defects, due to mantle cell lymphoma, prolymphocytoid variant, with marked leukocytosis, $1227 \times 10^{9} / \mathrm{l}$. He had splenomegaly but no lymphadenopathy or hepatomegaly. The tumor cells were CD5 +, CD19+, CD20 +, FMC$7+$, and kappa light chain restricted. Immunohistochemistry showed expression of p53 and of cyclin D1. Fluorescent in situ hybridization demonstrated $t(11 ; 14)$ with translocation between CYCLIN D1 and the immunoglobulin heavy-chain genes. The patient received leukapheresis and aggressive chemotherapy, but the leukocyte count remained above $100 \times 10^{9} /$. The patient's condition rapidly deteriorated with lymphomatous infiltration of his lungs and soft tissues, and he expired 6 months after diagnosis. While it is known that mantle cell lymphoma may have a leukemic phase, the degree of leukocytosis in this case exceeds that previously reported in the literature and resulted in a clinical syndrome of leukostasis. Modern Pathology (2004) 17, 879-883. doi:10.1038/modpathol.3800139
\end{abstract}

Keywords: mantle cell lymphoma; prolymphocytoid variant; leukostasis syndrome

The recent World Health Organization (WHO) classification defines mantle cell lymphoma as a distinct entity characterized by a unique immunophenotype (CD5 + , CD19 +, CD20 +, FMC-7 +, bcl$2+$, cyclin $\mathrm{D} 1+$ ) and molecular translocation between the CYCLIN D1 gene on chromosome 11 and the immunoglobulin heavy-chain gene on chromosome 14, $t(11 ; 14)(q 13 ; q 32) .{ }^{1}$ While most cases are composed of small- to medium-sized lymphoid cells resembling centrocytes, the WHO recognizes four morphologic variants, including two blastoid forms (classic and pleomorphic) and variants resembling small lymphocytic or marginal zone B-cell lymphoma. The medical literature has reports of another variant termed prolymphocytoid or nucleolated..$^{2-5}$ The identification of mantle cell lymphoma is important because of its aggressive clinical course. It is incurable with standard treatment regimens and has a median survival of only $3-5$ years. $^{6-8}$

Correspondence: Dr JC Mattson, MD, Department of Clinical Pathology, William Beaumont Hospital, 3601 W 13 Mile Road, Royal Oak, MI 48073, USA.

E-mail: jmattson@beaumont.edu

Received 19 December 2003; accepted 29 January 2004
Reports of mantle cell leukemia with prolymphocytoid or nucleolated morphology, as seen in the current case, are rare. ${ }^{2-5}$ We are aware of only one prior report describing mantle cell lymphoma with a leukocyte count over $300 \times 10^{9} / 1\left(689 \times 10^{9} / 1\right) .{ }^{4}$ Here, we describe a case of mantle cell lymphoma, prolymphocytoid variant, with marked leukocytosis of $1227 \times 10^{9} / \mathrm{l}$ and with a leukostasis syndrome.

\section{Case presentation}

A 76-year-old man presented to his ophthalmologist with a 4-month history of oculodynia, blurred vision, and weight loss of $4.5 \mathrm{~kg}$. He had no fever, chills, or diaphoresis. A complete blood count revealed leukocytosis of $1227 \times 10^{9} / \mathrm{l}$ with $98 \%$ lymphocytes (Table 1). His past medical history and family history were unremarkable. Physical examination revealed a visual field defect peripherally. His lungs were clear to auscultation. He had splenomegaly but no adenopathy, hepatomegaly, or skin lesions. Additional blood studies showed a positive direct Coombs, but haptoglobin and bilirubin were normal.

Computerized tomography scan of the chest, abdomen, and pelvis revealed mild splenomegaly 
without focal lesions. There were interstitial changes in the lung bases along with a left pleural effusion. There was no lymphadenopathy. A magnetic resonance imaging study of the brain was unremarkable.

A bone marrow biopsy was performed. The bone marrow morphology, flow cytometry, immunohistochemistry, and cytogenetic FISH analysis were consistent with mantle cell lymphoma, prolymphocytoid variant

Owing to the leukostasis syndrome, the patient was treated with daily leukapheresis for 1 week. The leukocyte count dropped to $670 \times 10^{9} / \mathrm{l}$, resulting in symptomatic relief. The leukapheresis was followed by chemotherapy with hyperCVAD (cyclophosphamide, doxorubicin, vincristine and dexamethasone) alternating with methotrexate and cytosine arabinoside. After two cycles of chemotherapy, the leukocyte count dropped to $415 \times 10^{9} / 1$, and a postchemotherapy bone marrow biopsy showed persistent extensive involvement by mantle cell lymphoma. The patient's leukocyte count never dropped below $100 \times 10^{9} /$ l (Figure 1 ).

Table 1 Laboratory findings at presentation

\begin{tabular}{lcc}
\hline Blood test & Result & Normal range \\
\hline WBC $\left(10^{9} / \mathrm{l}\right)$ & 1227 & $4.4-10.1$ \\
$\quad$ Differential $\left(10^{9} / \mathrm{l}\right)$ & $1208(98.5 \%)$ & $1.1-4.5$ \\
Lymphocytes & $12.3(1.0 \%)$ & $0.0-0.9$ \\
Monocytes & $6.1(0.5 \%)$ & $2.0-6.2$ \\
$\quad$ Neutrophils & 2.29 & $4.31-5.48$ \\
Red blood cell count $\left(10^{12} / \mathrm{l}\right)$ & 72 & $134-170$ \\
Hemoglobin $(\mathrm{g} / \mathrm{l})$ & 65 & $25-85$ \\
Reticulocyte count $\left(10^{9} / \mathrm{l}\right)$ & 142 & $140-413$ \\
Platelet count $\left(10^{9} / \mathrm{l}\right)$ & 19 & $5.1-21$ \\
Bilirubin $(\mu \mathrm{mol} / \mathrm{l})$ & 5.4 & $2.9-7.9$ \\
Blood urea nitrogen $(\mathrm{mmol} / \mathrm{l})$ & $3+(\mathrm{IgG}$ only) & Negative \\
Direct Coombs test & 0.75 & $0.60-2.70$ \\
Haptoglobin $(\mathrm{g} / \mathrm{l})$ & 639 & $100-238$ \\
Lactate dehydrogenase $(\mathrm{U} / \mathrm{l})$ & 80 & $62-133$ \\
Serum creatinine $(\mu \mathrm{mol} / \mathrm{l})$ & & \\
\hline
\end{tabular}

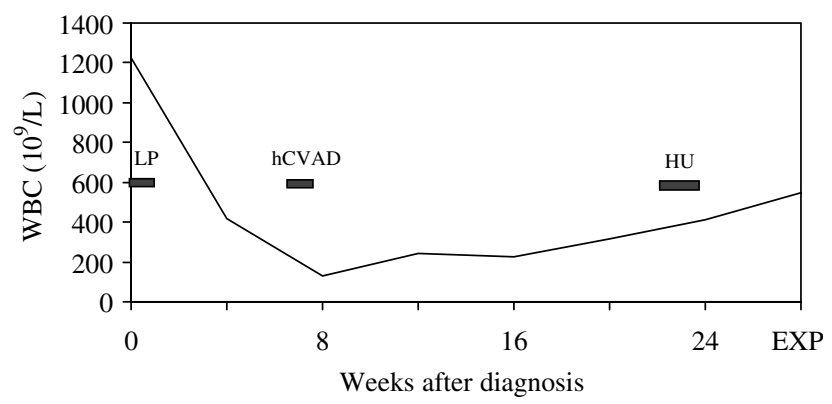

Figure 1 White blood cell count (WBC) over time. The patient received leukapheresis for 1 week after diagnosis. Leukapheresis was followed immediately by hyperCVAD chemotherapy that ended during week 7. Hydroxyurea was administered during weeks 22-24. The patient expired 6 months after diagnosis.
The patient's clinical course was complicated by a fever that subsided with antibiotics, and deep venous thrombosis of the left upper arm that required anticoagulation therapy. Supportive therapy, including hydroxyurea and three additional treatments of leukapheresis, was provided over the following months, but this was not effective in further reducing the leukocyte count. The patient developed a deep thigh mass and a symptomatic pleural effusion that showed involvement by lymphoma on fine-needle aspiration and thoracentesis, respectively. He died 6 months after diagnosis. No autopsy was performed.

\section{Materials and methods}

\section{Morphology}

The peripheral blood was stained with WrightGiemsa and bone marrow aspirate smears were stained with Jenner-Giemsa. The bone marrow aspirate clot and needle core biopsy were fixed in B5, embedded in paraffin, and stained with hematoxylin and eosin.

\section{Flow Cytometry}

Three-color flow cytometry was performed on the peripheral blood and bone marrow aspirate using a FACScan (Becton, Dickinson, and Company, San Jose, CA, USA). Briefly, leukocytes were isolated from EDTA anticoagulated peripheral blood or marrow by centrifugation with $0.1 \%$ bovine serum albumin in phosphate-buffered saline and were incubated with antibodies directed to the following antigens: CD2, CD3, CD4, CD5, CD7, CD8, CD10, CD11c, CD16, CD19, CD20, CD23, CD34, CD38, CD45, CD56, CD64, FMC-7, and kappa and lambda immunoglobulin light chains. Listmode files were analyzed with WinList (Verity Software House, Topsham, ME, USA).

\section{Immunohistochemistry}

Immunohistochemical stains were performed on the aspirate clot and bone marrow core biopsy using an Autostainer (DakoCytomation, Inc., Carpinteria, CA, USA). Sections were deparaffinized in xylene and alcohol. Antigen retrieval for CD5, CD10, CD20, CD23, CD45, bcl-2, p53 (DO-7) was accomplished by steaming the paraffin sections in EDTA buffer for 30 min using a commercial vegetable steamer. Antigen retrieval for cyclin D1 (DCS-6) was performed by steaming sections in citrate buffer for $25 \mathrm{~min}$. Detection was with the avidin-biotin peroxidase complex. 


\section{Fluorescent In Situ Hybridization}

Fluorescent in situ hybridization (FISH) was performed on interphase cells using LSI IgH/CCND1 dual color, dual fusion DNA probes (Vysis, Inc., Downers Grove, IL, USA). One probe labeled with SpectrumGreen hybridized to the immunoglobulin heavy-chain region at $14 q 32$, and another probe labeled with SpectrumOrange hybridized to the CYCLIN D1 (CCND1) gene at 11q13. The $\mathrm{t}(11 ; 14)(\mathrm{q} 13 ; \mathrm{q} 32)$ would produce a yellow fusion signal due to juxtaposition of the green and orange fluorescence. Bone marrow aspirate slides were prepared for the FISH analysis according to the manufacturer's protocol. A total of 200 interphase cells were scored.

\section{Pathology}

The peripheral blood smear showed marked leukocytosis of small- to medium-sized mature lymphocytes with coarse chromatin and with slightly irregular nuclear contours (Figure 2). Many of the tumor cells had one or more prominent nucleoli. There were occasional cytoplasmic blebs and rare free cytoplasmic fragments. Lymphocytes with similar morphology accounted for $86 \%$ of nucleated cells in the bone marrow aspirate smears. The bone marrow biopsy was markedly hypercellular (>95\%) due to a diffuse lymphoid infiltrate.

Immunophenotyping by flow cytometry revealed a discrete lymphoid population (bright CD45 and low side scatter) that expressed CD5, CD19, moderate intensity CD20, FMC-7, and moderate intensity monotypic kappa light chain. Immunohistochemistry on paraffin-embedded tissues showed strong staining for CD20, p53 (>80\% of cells) and bcl-2. Staining was positive focally for cyclin D1. CD5, CD23, and CD45RO were negative by immunohistochemistry. FISH on the marrow aspirate identified the $\mathrm{t}(11 ; 14)(\mathrm{q} 13 ; \mathrm{q} 32)$ between the immunoglobulin heavy-chain gene and CYCLIN D1 gene in 197 cells out of 200 (Figure 2).

\section{Discussion}

We report a case of mantle cell lymphoma, prolymphocytoid variant, presenting with marked leukocytosis and with a leukostasis syndrome, including oculodynia, blurred vision, and visual field defects.

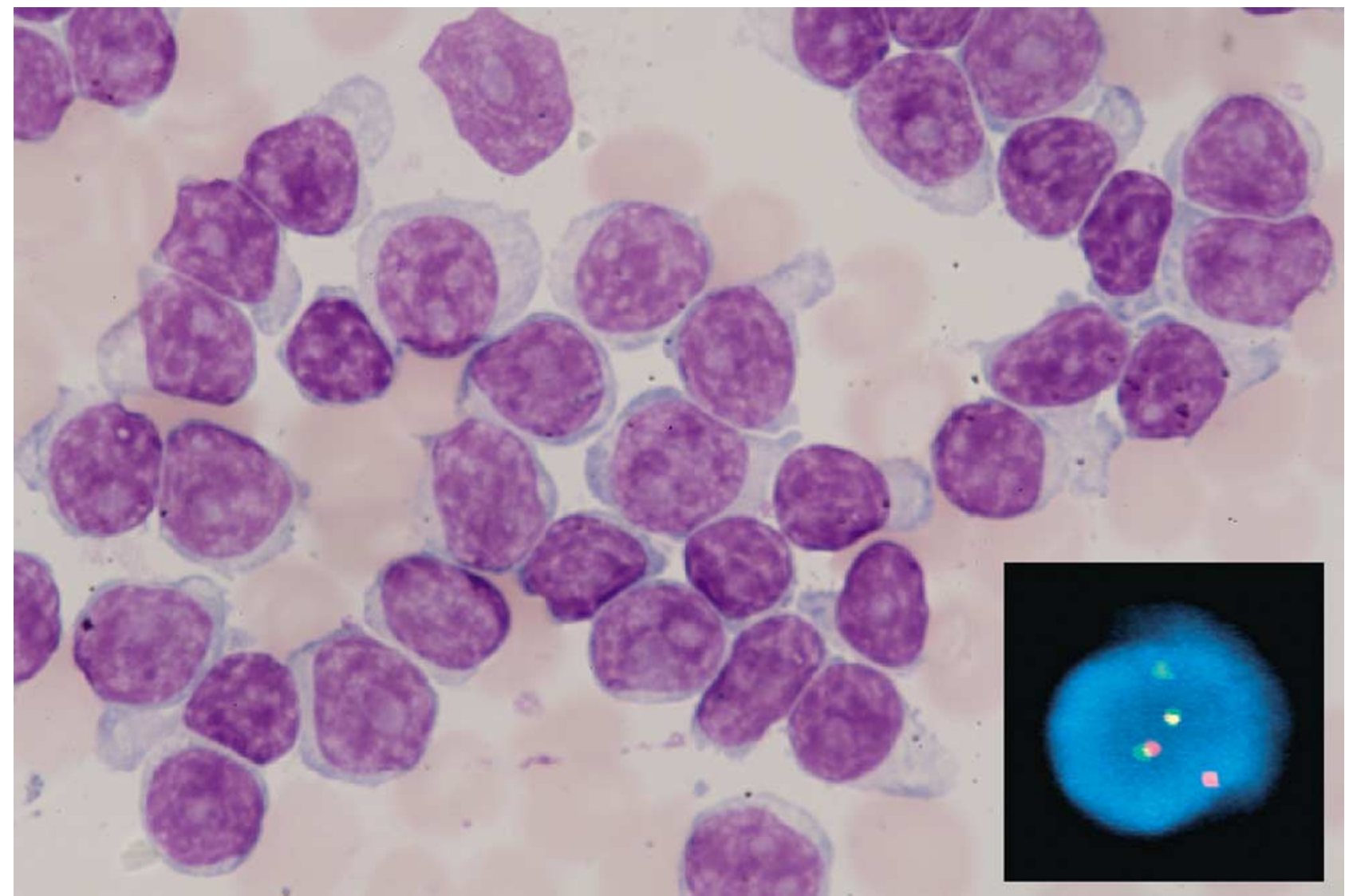

Figure 2 Wright-Giemsa-stained peripheral blood smear (original magnification $\times 2000$ ). Inset is FISH performed on interphase cells using one probe for the immunoglobulin heavy-chain region at 14q32 labeled with SpectrumGreen and another probe for the CYCLIN D1 (CCND1) gene at 11q13 labeled with SpectrumOrange. Juxtaposition of green and orange fluorescence produces yellow fusion signals in the cell nucleus. 
To our knowledge, the absolute lymphocyte count of $1227 \times 10^{9} / 1$ is the highest level reported in the medical literature for mantle cell lymphoma. The diagnosis was confirmed by morphology and by ancillary studies. Immunophenotyping by flow cytometry was consistent with mantle cell lymphoma. Immunohistochemistry showed expression of cyclin D1. FISH demonstrated the $t(11 ; 14)$ translocation between the immunoglobulin heavy-chain gene and CYCLIN D1 gene.

Prior reports have described mantle cell lymphoma involving the peripheral blood, although the amount of involvement varies in the medical literature. Cohen et al ${ }^{9}$ identified leukemic lymphoma cells in $77 \%(27 / 35)$ of patients with mantle cell lymphoma, but most of these cases did not have an absolute lymphocytosis at diagnosis. Other studies indicate that overt involvement of peripheral blood is less common (10-27\%). ${ }^{1,10,11}$ The patient reported here had marked leukocytosis causing leukostasis syndrome, which is a rare occurrence in mantle cell lymphoma.

Most patients with mantle cell lymphoma present with lymphadenopathy, ${ }^{1}$ but recent studies have noted that some patients present with marked leukocytosis, marrow involvement, and splenomegaly but no lymphadenopathy, similar to the current case. The latter cases were sometimes misdiagnosed as prolymphocytic leukemia in the older medical literature. Mantle cell lymphoma and prolymphocytic leukemia may have similarities in clinical presentations, microscopic features, and flow cytometric immunophenotypes. Mantle cell lymphoma is distinguished by increased cyclin D1 resulting from the $\mathrm{t}(11 ; 14)$ translocation between the immunoglobulin heavy-chain and CYCLIN D1 genes. Prolymphocytic leukemia lacks these features.

The current case was classified as mantle cell lymphoma, prolymphocytoid variant. There have been four prior reports of prolymphocytoid or nucleolated mantle cell lymphoma. ${ }^{2-5}$ The previously reported cases had the $t(11 ; 14)$ by either conventional cytogenetics or FISH. Mantle cell lymphoma had a characteristic immunophenotype: CD5 +, CD19 + , and CD20 + , and usually negative for CD23. Cyclin D1 was increased in 97\% (28/29), and FMC-7 was positive in $89 \%$ (24/27). One study reported a worse overall survival for a large/blastoid morphologic variant compared to a small-cell type. ${ }^{4}$ Clinical follow-up in all four studies ranged from 1 to 126 months with three patients alive at the time of publication. The current patient died 6 months after diagnosis.

The current patient's high tumor burden is a contributing factor to the aggressiveness of this leukemic presentation. Leukostasis syndromes may be associated with headache, confusion, tinnitus, papilledema, ataxia, obtundation, dyspnea, hypoxemia, metabolic abnormalities, coagulopathy, and tumor lysis syndrome. ${ }^{12}$ The current patient presented with leukocytosis of $1227 \times 10^{9} / \mathrm{l}$, with oculodynia, blurred vision, and visual field defects. The patient's symptoms improved with leukapheresis. The combination of leukapheresis and highdose chemotherapy reduced the leukocyte count but not below $100 \times 10^{9} / \mathrm{l}$.

The poor outcome in this case may be linked not only to the aggressive morphologic subtype of mantle cell lymphoma and to the leukocytosis but also to abnormal p53. The marrow biopsy had increased p53 protein. Wild-type p53 acts as a tumor suppressor gene that can induce growth arrest and/or apoptosis when DNA mutations occur in the cell. However, p53 mutations can inhibit this important function, allowing the cell to undergo mitosis in the presence of genetic defects. ${ }^{13-15}$ Although p53 mutations are rare in small B-cell lymphomas, up to half of high-grade B-cell lymphomas have been shown to overproduce p53 protein, presumably due to p53 gene mutations resulting in abnormal stabilization of the protein. ${ }^{16}$ In addition, p53 mutations have been found in high grade transformation of low-grade lymphomas. ${ }^{17-19}$ Some studies have found that p53 mutations and/or overproduction in mantle cell lymphoma are associated with aggressive variants, including large cell or blastic types. ${ }^{20-22}$ Wong et $a l^{5}$ found overexpression of p53 in one of four nucleolated mantle cell lymphomas. These findings support the hypothesis that mutations of p53 or other genes involved in cell cycle regulation are associated with transformation of typical mantle cell lymphoma to an aggressive type. $^{23}$

In conclusion, we present a case of mantle cell lymphoma with aggressive features: prolymphocytoid morphology, p53 overproduction, marked leukocytosis, and leukostasis syndrome. It is likely that these biological features played an important role in the rapid clinical course. This case underscores the need for new therapeutic approaches for treating patients with mantle cell lymphoma.

\section{References}

1 Swerdlow SH, Berger F, Isaacson PI, et al. Mantle cell lymphoma. In: Jaffe ES, Harris NL, Stein H, Vardiman JW (eds). World Health Organization Classification of Tumours. Pathology and Genetics of Tumours of Haematopoietic and Lymphoid Tissues. International Agency for Research on Cancer (IARC) Press: Washington, DC, 2001, pp 168-170.

2 Nelson BP, Variakojis D, Peterson LC. Leukemic phase of B-cell lymphomas mimicking chronic lymphocytic leukemia and variants at presentation. Mod Pathol 2002;15:1111-1120.

3 Schlette E, Bueso-Ramos C, Giles F, et al. Mature B-cell leukemias with more than $55 \%$ prolymphocytes. A heterogeneous group that includes an unusual variant of mantle cell lymphoma [comment]. Am J Clin Pathol 2001;115:571-581. 
4 Schlette E, Lai R, Onciu M, et al. Leukemic mantle cell lymphoma: clinical and pathologic spectrum of twenty-three cases. Mod Pathol 2001;14:1133-1140.

5 Wong KF, So CC, Chan JK. Nucleolated variant of mantle cell lymphoma with leukemic manifestations mimicking prolymphocytic leukemia [comment]. Am J Clin Pathol 2002;117:246-251.

6 Weisenburger DD, Armitage JO. Mantle cell lymphoma -an entity comes of age. Blood 1996;87: 4483-4494.

7 Armitage JO, Weisenburger DD. New approach to classifying non-Hodgkin's lymphomas: clinical features of the major histologic subtypes. Non-Hodgkin's Lymphoma Classification Project. J Clin Oncol 1998; 16:2780-2795.

8 Weisenburger DD, Vose JM, Greiner TC, et al. Mantle cell lymphoma. A clinicopathologic study of 68 cases from the Nebraska Lymphoma Study Group. Am J Hematol 2000;64:190-196.

9 Cohen PL, Kurtin PJ, Donovan KA, et al Bone marrow and peripheral blood involvement in mantle cell lymphoma. Br J Haematol 1998;101:302-310.

10 Argatoff LH, Connors JM, Klasa RJ, et al. Mantle cell lymphoma: a clinicopathologic study of 80 cases. Blood 1997;89:2067-2078.

11 Pittaluga S, Verhoef G, Criel A, et al. Prognostic significance of bone marrow trephine and peripheral blood smears in 55 patients with mantle cell lymphoma. Leuk Lymphoma 1996;21:115-125.

12 Porcu P, Farag S, Marcucci G, et al. Leukocytoreduction for acute leukemia. Ther Apher 2002;6:15-23.
13 Hunter T, Pincs J. Cyclins and cancer. II: cyclin D and CDK inhibitors come of age. Cell 1994;79:573-582.

14 Sherr CJ. Cancer cell cycles. Science 1996;274:1672-1677.

15 Sherr CJ. The Pezcoller lecture: cancer cell cycles revisited. Cancer Res 2000;60:3689-3695.

16 Imamura J, Miyoshi I, Koeffler HP. p53 in hematologic malignancies. Blood 1994;84:2412-2421.

17 Ichikawa A, Hotta T, Takagi N, et al. Mutations of p53 gene and their relation to disease progression in B-cell lymphoma. Blood 1992;79:2701-2707.

18 Sander CA, Yano T, Clark HM, et al. p53 mutation is associated with progression in follicular lymphomas. Blood 1993;82:1994-2004.

19 Lo Coco F, Gaidano G, Louie DC, et al. p53 mutations are associated with histologic transformation of follicular lymphoma. Blood 1993;82:2289-2295.

20 Zoldan MC, Inghirami G, Masuda Y, et al. Large-cell variants of mantle cell lymphoma: cytologic characteristics and p53 anomalies may predict poor outcome. $\mathrm{Br}$ J Haematol 1996;93:475-486.

21 Pinyol M, Hernandez L, Cazorla M, et al. Deletions and loss of expression of p16INK4a and p21Waf1 genes are associated with aggressive variants of mantle cell lymphomas. Blood 1997;89:272-280.

22 Hernandez L, Fest T, Cazorla M, et al. p53 gene mutations and protein overexpression are associated with aggressive variants of mantle cell lymphomas. Blood 1996;87:3351-3359.

23 Swerdlow SH, Williams ME. From centrocytic to mantle cell lymphoma: a clinicopathologic and molecular review of 3 decades. Hum Pathol 2002;33:7-20. 